Radial and Nonradial Pulsations as Probes of Stellar Physics

ASP Conference Series, Vol. 259, 2002

C. Aerts, T.R. Bedding, \& J. Christensen-Dalsgaard, eds.

\title{
Primary Observational Distributions of $\delta$ Scuti Variables
}

\author{
E. Rodríguez \\ Instituto de Astrofísica de Andalucía, CSIC, P.O. Box 3004, E-18080 \\ Granada, Spain
}

M. Breger

Institut für Astronomie, Universität Wien, Türkenschanzstr. 17, A-1180, Austria

The distribution of the known $\delta$ Scuti-type variables (R00 catalogue, Rodriguez et al., 2000) is analysed on the basis of some primary observational properties such as visual amplitude, period, visual magnitude and rotational velocities. The present distributions are also compared with those resulting from the earlier catalogue by Rodríguez et al. (1994, R94). The contributions from the Hipparcos, OGLE and MACHO long-term monitoring projects are also discussed. These contributions are quite different in the $\mathrm{N}-\Delta \mathrm{V}$ and $\mathrm{N}-\mathrm{V}$ diagrams, in the sense that the variables discovered by the Hipparcos satellite are much brighter and with shorter periods than those discovered by the OGLE and MACHO projects.

Concerning the $\mathrm{N}-\Delta \mathrm{V}$ diagram, we find that the gap, which was evident in the R94 catalogue for variables with visual amplitudes between $0 .^{m} 1$ to $0 .^{m} 3$, has disappeared. Thus, it seems that there is not a strict separation in two groups relative to the amplitude (low amplitude for variables with $\Delta V<0{ }^{m} 1$ and high amplitude for variables with $\Delta \mathrm{V}>0 .{ }^{m} 3$ ) for the $\delta$ Scuti-type pulsators.

Concerning the $\langle\Delta \mathrm{V}\rangle-\mathrm{P}$ distribution, the selection effect "the amplitude is larger when the period is longer" still remains, but it is very small as compared to the R94 catalogue. In fact, this selection effect does not exist in the variables discovered by Hipparcos and MACHO and it is very small (if any) in the new variables which come from OGLE.

Concerning the $\mathrm{N}-\mathrm{V}$ and $\langle\Delta \mathrm{V}\rangle-\mathrm{V}$ distributions, the selection effect "the amplitude is larger when the star is fainter" still remains, but it is much smaller than in the R94 catalogue. Nevertheless, when we analyse each contribution separately, we can see that this selection effect is small, but still remains.

Concerning the $\mathrm{N}-v \sin i$ plot, the distribution is presently more smoothed and the stars seem to be uniformly distributed in the range for rotational velocities below than $180 \mathrm{~km} \mathrm{~s}^{-1}$. Similarly to R94, the variables displaying large amplitude are very slow rotators. This is confirmed when we plot the contributions from the variables with low $\left(\Delta V \leq 0 .{ }^{m} 03\right)$ and large $\left(\Delta V \geq 0 .^{m} 1\right)$ amplitudes. In fact, the mean value of $v \sin i$ for $\delta$ Sct variables with $\Delta V \leq 0 .^{m} 03$ is found to be of $109( \pm 58) \mathrm{km} \mathrm{s}^{-1}$, whereas this is much smaller for the large amplitude pulsators $(<v \sin i\rangle=22( \pm 10) \mathrm{km} \mathrm{s}^{-1}$ for the variables with $\Delta V \geq 0{ }^{m} 1$ and a very similar value is found for the variables with $\Delta V \geq 0 .^{m} 3$ ). This agrees well when 
considering the parametric resonance as a mechanism to limit the amplitude of the pulsations, as mentioned in R94.

\section{References}

Rodríguez, E., López de Coca, P., Rolland, A., Garrido, R., \& Costa, V. 1994, A\&AS, 106, 21

Rodríguez, E., López-González, M. J., \& López de Coca, P. 2000, A\&AS, 144, 469 